verwendeten Gasproben wurde durch Messung der Dampfdruckdifferenzen zwischen Proben verschiedener Fraktionen der Desorption und verschiedener Herstellungsgänge kontrolliert.

Für die Umrechnung der gemessenen Dampfdruckdifferenzen auf die der reinen isotopen Komponenten wurde die Gültigkeit des Raoultschen Gesetzes über den ganzen Mischungsbereich vorausgesetzt.

Aus den Messungen ergeben sich Temperaturfunktionen für das Dampfdruckverhältnis, die sich durch folgende allgemeine Gleichung darstellen lassen:

$$
\log p_{\mathrm{L}} / p_{\mathrm{S}}=A J T+B \lg T-C .
$$

In Tab. 1 sind die Konstanten $A, B, C$ und Werte für $p_{\mathrm{L}} / p_{\mathrm{S}}$ am Tripelpunkt für $\mathrm{CO}$ und $\mathrm{CH}_{4}$ angegeben.

\begin{tabular}{|c|c|c|c|c|}
\hline & $\boldsymbol{A}$ & $B$ & $C$ & $\left(\mathrm{p}_{\mathrm{L}} / \mathrm{p}_{\mathrm{s}}\right)_{\text {Tripelpunkt }}$ \\
\hline CO & 1,2190 & 0,018692 & 0,047251 & $1,0113 \pm 0,0002$ \\
\hline $\mathrm{CH}_{4}$ & 4,19855 & 0,077507 & 0,19588 & $1,0049 \pm 0,0002$ \\
\hline
\end{tabular}

Tab. 1.

In Tab. 2 sind von uns gemessene Werte für $\left(p_{\mathrm{L}} / p_{\mathrm{S}}\right)_{\mathrm{CO}}$ Werten gegenübergestellt, die aus einer von Kronberger ${ }^{2}$ angegebenen empirischen Gleichung berechnet sind.

\section{Über einige Isotope des Technetiums}

Von J. Flegenheimer und W. Seelmann-Eggebert

Laboratorios de Radioquímica de la Comisión Nacional de la Energia Atómica, Buenos Aires

(Z. Naturforschg. 9 a, 806 [1954]; eingeg. am 14. August 1954]

Die bisher nicht bekannte Halbwertszeit der Tochtersubstanz des unter den Kernspaltprodukten aufgefundenen Molybdänisotops mit einer Halbwertszeit von etwa 11 Min. (102 ?) wurde gemessen und beträgt $5 \pm 1$ Sek.

Zur Halbwertszeitbestimmung wurde Spaltmolybdän radiochemisch abgetrennt, gereinigt und zuletzt als Bleimolybdat gefällt. Der Niederschlag wurde in einer Mischung von Salz- und Weinsäure gelöst, Perrhenationen als Technetiumträger zugegeben und mit Tetraphenylarsoniumchlorid gefällt. In 6 Sek. gelang es, Rhenium zu fällen, den Niederschlag zu filtrieren und zur Messung zu bringen.

Als Meßgerät wurde ein besonders zur Bestimmung kurzer Halbwertszeiten umgebauter Integrator verwendet, mit dem man den Ladungsanstieg im Kondensator in Zeitintervallen registriert, die so klein gegen die Zeitkonstante sind, daß der Ausgleichsstrom durch den Entladungswiderstand noch keine Rolle spielt. Die Apparatur wurde von Herrn Dr. K. Fraenz (Comisión Nacional de la Energía Atómica) erdacht und entwickelt.

Auch der Abfall des Spaltmolybdäns wurde mit einer Absorptionsfolie, welche die $\beta$-Strahlen des Systems der Isobarenreihe mit der Massenzahl 101 völlig unterdrückt, gemessen. Nach Abzug der $\gamma$-Aktivität der Isobarenreihe 101 ergab sich eine Halbwertszeit für die Muttersubstanz des 5 Sek.-Technetiums von

\begin{tabular}{|c|c|c|}
\hline$T$ & $\begin{array}{c}\left(p_{\mathrm{L}} / p_{\mathrm{s}}\right)_{\mathrm{co}} \\
\text { diese Arbeit }\end{array}$ & $\begin{array}{c}\left(p_{\mathrm{L}} / p_{\mathrm{s}}\right)_{\mathrm{co}} \\
\text { Kronberger }\end{array}$ \\
\hline 69,28 & $1,0109_{0}$ & $1,0109_{2}$ \\
69,97 & $1,0106_{8}$ & $1,0107_{0}$ \\
71,53 & $1,0101_{9}$ & $1,0102_{3}$ \\
72,49 & $1,0100_{1}$ & $1,0099_{7}$ \\
74,43 & $1,0094_{6}$ & $1,0094_{5}$ \\
\hline
\end{tabular}

Tab. 2.

Die theoretische Berechnung des Dampfdruckverhältnisses $p_{{ }^{12} \mathrm{CH}_{4}} / p^{{ }^{13} \mathrm{CH}_{4}}$ am Tripelpunkt läßt sich analog zu der Berechnung von $p^{20} \mathrm{Ne} / p^{22} \mathrm{Ne}$ durch Keesom und Haantjes ${ }^{3}$ durchführen. Die prozentuelle Abweichung vom experimentellen Wert hat dieselbe Größe und Richtung.

Im Falle des Kohlenoxyds ist die theoretische Berechnung weit komplizierter und ihr Endergebnis ungenauer.

Trotzdem läßt sich auch auf Grund dieser Rechnung für das Koblenoxyd am Tripelpunkt ein größerer relativer Dampfdruckunterschied zwischen beiden isotopen Verbindungen erwarten als für Methan.

${ }^{2}$ Siehe T. F. Johns u. H. London, A. E. R. E. Report, Harwell 1951.

${ }^{3}$ Keesom u. J. Haantjes, Physica 2, 986 [1935].

$11,5 \pm 0,5$ Min., welche mit den Bestimmungen anderer Autoren gut übereinstimmt.

Bei der Bestrahlung von $\mathrm{RuO}_{2}$ mit schnellen Neutronen wurde durch eine n,p-Reaktion ein neues Technetiumisotop mit einer Halbwertszeit von $3,8 \pm 0,2$ Min. aufgefunden und isoliert. Bei diesem Nuklid handelt es sich vermutlich um ${ }^{104} \mathrm{Tc}$, da das zweite bisher noch unbekannte Technetiumisotop ${ }^{98} \mathrm{Tc}$ wahrscheinlich eine lange Halbwertszeit hat ${ }^{1}$.

Bei der chemischen Abtrennung wurde das bestrahlte Rutheniumoxyd mit Ammoniak, dem einige Tropfen Wasserstoffsuperoxyd zugesetzt waren, behandelt. Ein Teil des gebildeten Technetiums kann so mit der wässerigen Phase vom Ruthenoxyd durch einfache Filtration getrennt werden. Zum Filtrat wurden Rhenium- und Molybdän-Träger zugesetzt und das Technetium mit Tetraphenylarsoniumchlorid gefällt. Die chemische Trennung vom Ende der Bestrahlung bis zum Messungsbeginn der Technetium-Fraktion dauerte 5 bis 6 Min.

Die Bestrahlungen wurden mit einem Philips-Kaskadengenerator von 1,4 MV durchgeführt. Deuteronen wurden beschleunigt, Lithium diente als target. Für die Durchführung der Bestrahlung möchten wir Herrn W. Scheuer und seinen Mitarbeitern herzlich danken; wie auch Herrn A. H. W. Aten jr. für die liebenswürdige Überlassung des Tetraphenylarsoniumchlorids.

Die vollständige Arbeit wird in Kürze in den Publicaciones de la Comisión Nacional de la Energía Atómica Serie Química erscheinen.

1 J. K. Lerohl, M. L. Pool, D. N. Kundu u. R. A. House, Phys. Rev. 92, 934 [1954]. 